\title{
Editorial
}

\section{Advances in Antenna Array Processing for Radar 2016}

\author{
Hang Hu, ${ }^{1}$ Xuesong Wang, ${ }^{2}$ Michelangelo Villano, ${ }^{3}$ Ahmed Shaharyar Khwaja, ${ }^{4}$ \\ Jeich Mar, ${ }^{5}$ and Wenchong Xie ${ }^{6}$ \\ ${ }^{1}$ School of Electronics and Information Engineering, Harbin Institute of Technology, Harbin 150001, China \\ ${ }^{2}$ State Key Laboratory of Complex Electromagnetic Environment Effects on Electronics and Information System, \\ National University of Defense Technology, Changsha 410073, China \\ ${ }^{3}$ Microwaves and Radar Institute, German Aerospace Center (DLR), Oberpfaffenhofen, 82234 Wessling, Germany \\ ${ }^{4}$ Faculty of Engineering and Natural Sciences, Sabanci University, Tuzla, 34956 Istanbul, Turkey \\ ${ }^{5}$ Department of Communications Engineering, Yuan-Ze University, Chung-Li 32003, Taiwan \\ ${ }^{6}$ Key Research Laboratory, Wuhan Early Warning Academy, Wuhan 430019, China \\ Correspondence should be addressed to Hang Hu; huhang@hit.edu.cn
}

Received 25 September 2016; Accepted 26 September 2016

Copyright (c) 2016 Hang Hu et al. This is an open access article distributed under the Creative Commons Attribution License, which permits unrestricted use, distribution, and reproduction in any medium, provided the original work is properly cited.

RAP (Radar Array Processing) is a very active, open, and concerned topic in the field of radar. It is of great significance to promote the progress of radar theory and technology. Although the RAP has experienced five decades of research, it still represents a fascinating discipline with great development potential.

At present, the rapid development of advanced radar processing techniques is closely related to the RAP. The former includes STAP (Space-Time Adaptive Processing), MIMO (Multiple-Input Multiple-Output) radar, multichannel SAR (Synthetic Aperture Radar), adaptive detection, radar ECCM (Electronic Counter-Countermeasure), and so forth.

PAR (Phased Array Radar), MIMO radar, MIMO-PAR, and digital array radar are suitable for constituting the multichannel system due to their inherent antenna structure. Consequently, we could fully apply a variety of advanced RAP techniques to these radars.

So far, the RAP has obtained fruitful achievements in theory and algorithm respects. However, the research and development on application, system, engineering, implementation of hardware, and so on are still far from enough. And those are just what researchers and radar engineers are particularly concerned about.

The study on RAP should adopt a "systemic" point of view. In other words, it should not be regarded simply as a specific theory, algorithm, or technique issue but should be considered under a uniform radar system framework. That is, we should incorporate RAP into adaptive detection, parameter estimation (such as adaptive monopulse), and data processing (such as adaptive tracking); consequently, the capabilities of the RAP could be assessed in the whole system.

In this 2016 special issue, we have collected papers covering the following aspects of RAP research and development: parasitic array receiver for ISAR imaging of ship targets using coastal radar, robust adaptive beamforming using low-complexity steering vector estimation and a covariance matrix reconstruction algorithm, FPC-root algorithm for 2DDOA estimation in sparse array, and design of the microstrip reflect array antenna by optimizing the reflection phase curve.

We would like to thank all the authors for their professional contributions and all the reviewers for their time and effort. A special thank goes to Dr. U. Nickel for his constructive guidance.

Hang $\mathrm{Hu}$ Xuesong Wang Michelangelo Villano Ahmed Shaharyar Khwaja

Jeich Mar Wenchong Xie 


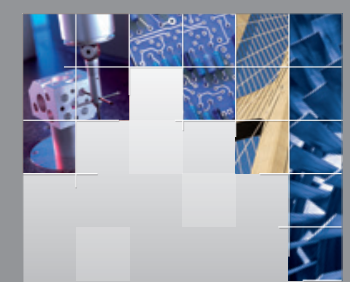

\section{Enfincering}
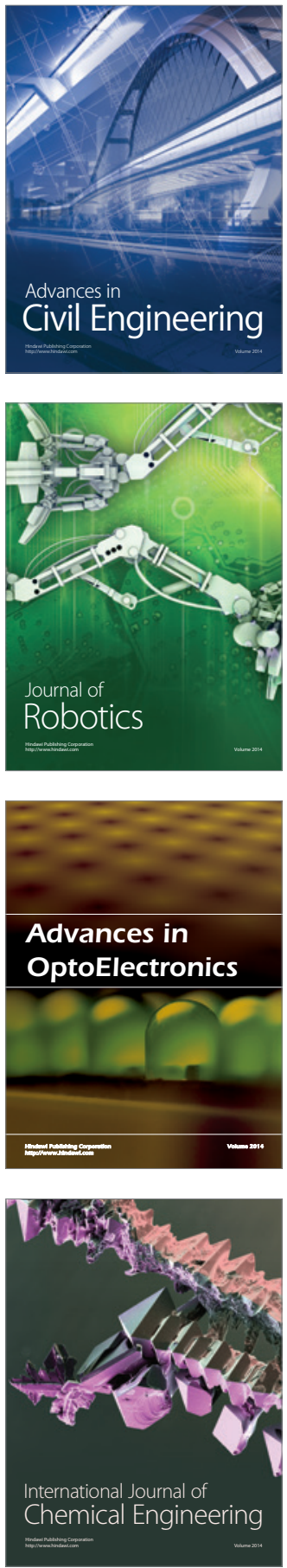

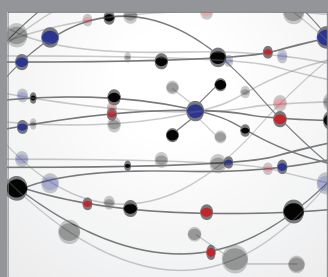

The Scientific World Journal

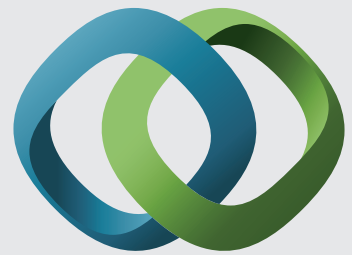

\section{Hindawi}

Submit your manuscripts at

http://www.hindawi.com
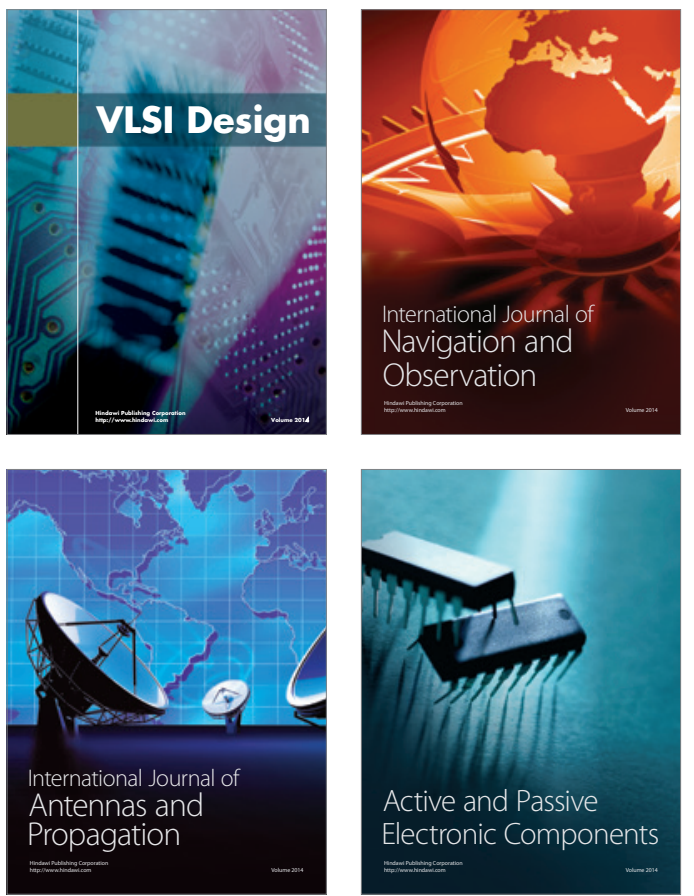
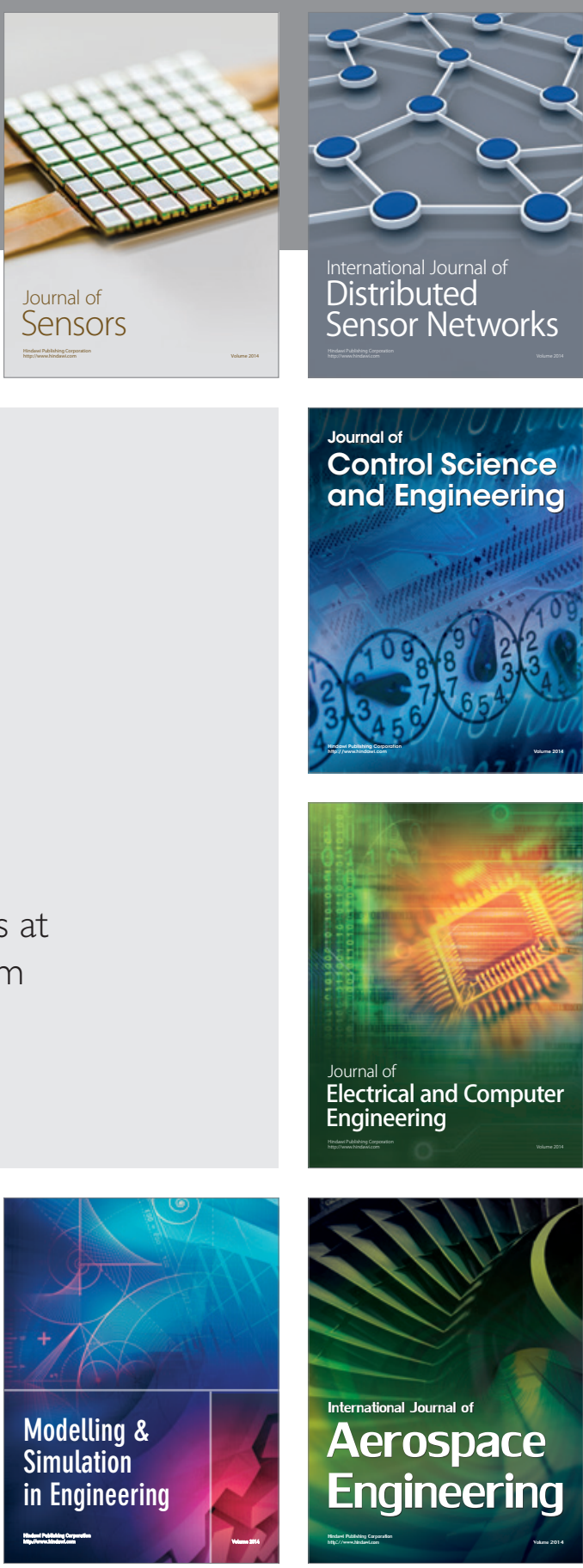

International Journal of

Distributed

Sensor Networks

Journal of

Control Science

and Engineering
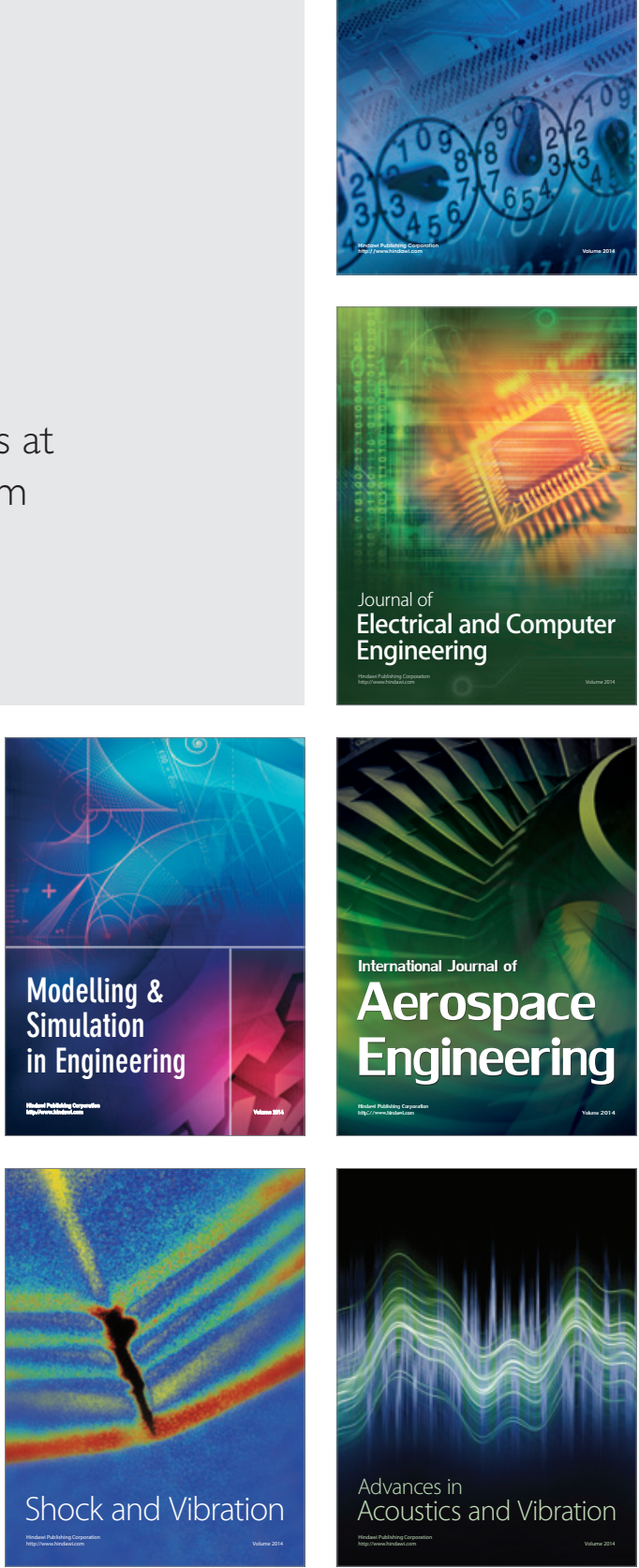